\title{
Medios rojos y espectadores azules: cuando las audiencias deciden emigrar. Selectividad ideológica de las audiencias ante la estatización de la información en Venezuela
}

\author{
Carmen Beatriz Fernández \\ Universidad de Navarra, España \\ carmenbeat@datastrategia.com
}

Recibido: 6/3/2018 / Aceptado: 19/3/2018

doi: 10.26439/contratexto2018.n029.1822

\begin{abstract}
Resumen. En Venezuela, el régimen populista-autoritario creó una nueva arquitectura comunicacional, en donde el esquema nacional de medios favoreció a la voz oficial. El empeño se consolidó en el 2013, al asumir Nicolás Maduro la presidencia tras la muerte de Hugo Chávez. Después de ese control hegemónico de los canales de medios, las audiencias venezolanas comenzaron a migrar a otros canales de medios no tradicionales, incluidos los digitales, que alcanzaron, para el caso venezolano, números muy altos. Este documento evalúa cómo las audiencias han ido migrando de plataformas informativas, en la medida en que el mensaje oficial emitido desde el gobierno iba tomando espacios; a la vez, resalta la importancia de los canales alternativos, basados, entre otras fuentes, en datos internos del canal de televisión oficial de la oposición en YouTube, así como en estadísticas y datos primarios de investigaciones de opinión pública.
\end{abstract}

Palabras clave: Venezuela / control de medios / medios de comunicación / audiencias / medios digitales 


\title{
Red Media and Blue Spectators: When Audiences Decide to Emigrate. Ideological Selectivity of the Audiences as a Reaction to the Nationalization of Information in Venezuela
}

\begin{abstract}
The populist-authoritarian regime in Venezuela created a new communicational architecture, in which the national media favored the government's narrative. Their efforts were consolidated in 2013, when Nicolás Maduro assumed the presidency after Hugo Chávez's death. After the governmental hegemonic control of media outputs, Venezuelan audiences started to emigrate toward non-traditional media outputs. These included digital media, which reached a high number of consumption. The following article evaluates how audiences have been emigrating from informational platforms, insofar as the official message emitted by the government began taking up an ever-increasing number of spaces. At the same time, this document highlights the importance of alternative channels based-among other sources- on internal data from the opposition's YouTube broadcasting channel, statistics and primary data from public opinion surveys.
\end{abstract}

Keywords: Venezuela / media control / mass media / audiences / digital media 


\section{Introducción}

E

ra el 18 de enero del 2017 y el presidente de Estados Unidos, Barack Obama, daba su última conferencia de prensa. Había gran expectativa al respecto, pues Obama era casi un rockstar por aquellos días. Siempre había sido un presidente medianamente popular, pero, en los últimos meses de su mandato, sus números de aprobación se dispararon unos diez puntos para cerrar el período con niveles de aprobación cercanos al 60 \% (Gallup, 2017), muy superiores a los que había alcanzado durante los ocho años de su gobierno, y también mayores que el promedio de los niveles de aprobación históricos de los presidentes salientes (Gallup, s. f.). Esa última conferencia de prensa fue emitida desde la Casa Blanca, transmitida en directo desde su canal oficial en YouTube y seguida en directo por un gran número de personas. La cuenta principal alcanzó picos cercanos a los 30000 espectadores en vivo, un número singularmente alto para la transmisión de un evento político, si bien muy inferior al récord histórico de 2 millones de personas que han llegado a ver transmisiones en vivo desde la plataforma durante los debates presidenciales de la última contienda del país del norte (YouTube, 2016).

Figura 1. Audiencia online en la última conferencia de prensa de Barack Obama

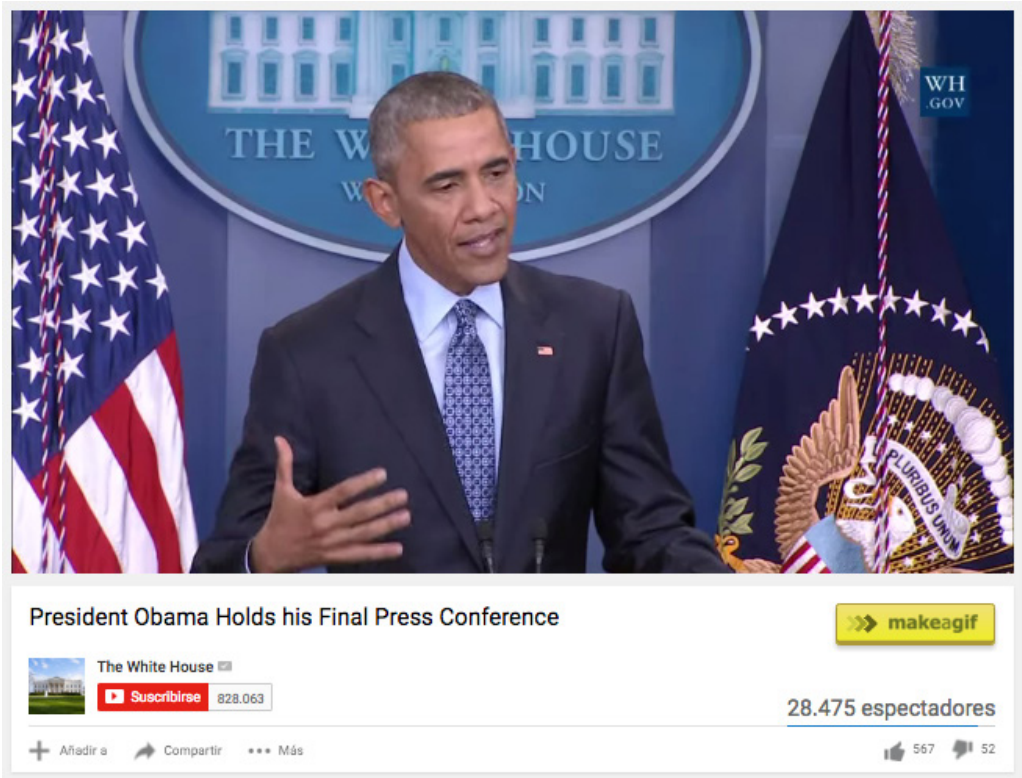

Fuente: Captura de pantalla (The [Obama] White House, 2017) 
Estados Unidos tiene una población de más de 300 millones de habitantes y una penetración de internet del 95,6 \% ${ }^{1}$ (Internet World Stats, s. f.); por ello, es fácil que todos los récords de audiencia en redes sociales caigan dentro de sus fronteras. Sin embargo, dos años antes, otro acontecimiento político había alcanzado picos inusitados para una transmisión en vivo: hasta 90000 personas $^{2}$ vieron en directo la transmisión del canal de Unidad Venezuela en YouTube, tras votar en las elecciones parlamentarias venezolanas del 2015. Venezuela tiene 10 veces menos población que Estados Unidos y una penetración de internet de solo el 60 \% (Internet World Stats, s. f.). ¿Cómo es posible, con tal disparidad de magnitudes, alcanzar cifras tan altas de audiencia en la plataforma YouTube?

Figura 2. Audiencia online tras las elecciones parlamentarias en Venezuela

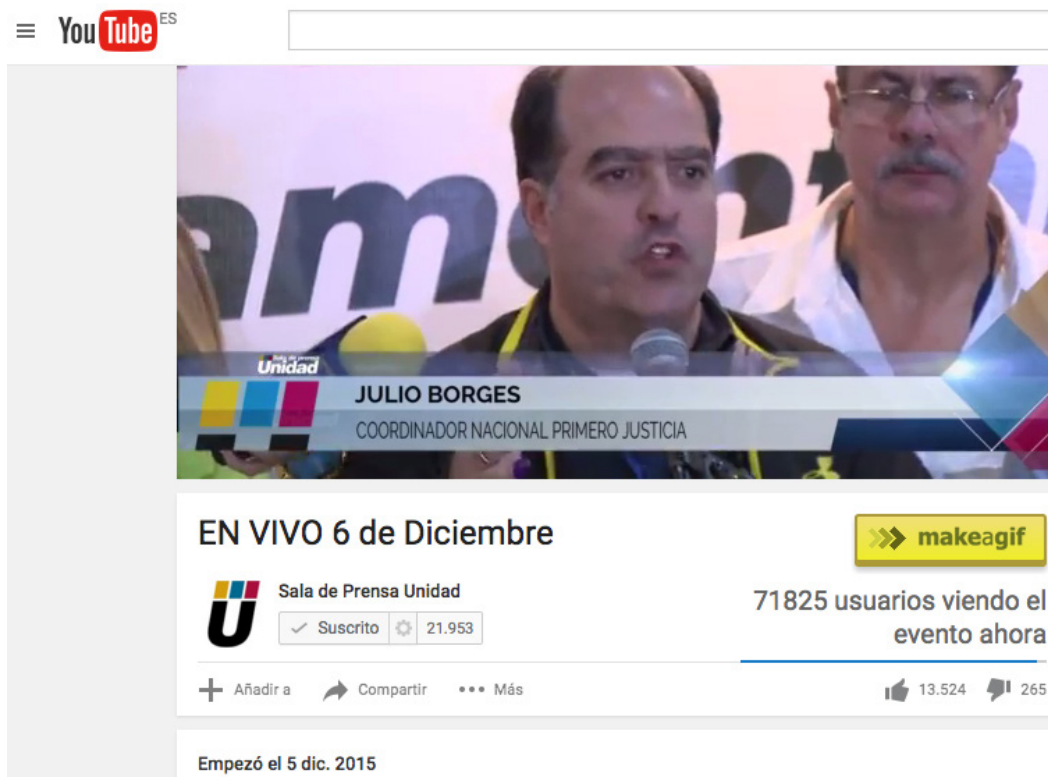

Fuente: Captura de pantalla (Sala de Prensa Unidad, 2015)

1 Datos hasta junio del 2017.

2 Entrevista realizada a la periodista María Fernanda Flores Mayorca, responsable del canal. 


\section{Medios rojos: ¿cómo llegamos hasta aquí?}

La elección presidencial venezolana de abril del 2013 condujo a un virtual empate entre los dos bloques políticos del país. Henrique Capriles Radonski había competido contra Nicolás Maduro, quien había sido ungido como heredero político tras el fallecimiento de Hugo Chávez. A partir de que Maduro asumiera la presidencia, se intensificaron los esfuerzos por lograr la "hegemonía comunicacional", que había sido declarada formalmente como un objetivo del gobierno de Chávez en el $2007^{3}$. El chavismo la consiguió casi al mismo tiempo en que comenzaba a ser minoría política. Habiendo en aquel 2013 un equilibrio político en el país, con dos segmentos de igual tamaño, una de las mitades fue silenciada en medios de comunicación por la nueva orientación editorial impuesta.

Durante el segundo semestre del 2013, en el inicio del gobierno de Nicolás Maduro, se consolidó una nueva arquitectura comunicacional (Fernández, 2014), con un esquema nacional de medios que favorecía de forma notoria el mensaje oficial. La autodenominada Revolución Bolivariana se ha identificado desde sus comienzos con el color rojo, y rojo fue el tono dominante en el entramado de medios de comunicación en Venezuela a partir de ese momento. Entre 1974 y el 2004, el Estado venezolano había contado con un canal de televisión pública: Venezolana de Televisión. De esa única señal televisiva oficial se pasó a tener seis. Para el 2013, el gobierno manejaba tres circuitos radiales con carácter nacional y más de 250 emisoras comunitarias. A ellos se sumaron tres diarios financiados con fondos públicos, así como varios medios privados con línea editorial alineada a la oficial. Todo ello configura un espacio de comunicaciones que a veces se ha denominado mixto-autoritario (Cañizales y Matos-Smith, 2015), pues permite la presencia del sector privado, pero forzando los contenidos y la línea editorial.

La consolidación del control del gobierno sobre los medios de comunicación se logró combinando la ocupación agresiva de los medios con un esfuerzo de adquisición. En varios casos, el régimen facilitó las condiciones y el acceso a las divisas preferenciales para que sectores empresariales afectos adquirieran medios de comunicación. Aquello fue lo sucedido con la televisora noticiosa Globovisión, con el muy popular diario de circulación nacional Últimas Noticias y con el histórico diario El Universal.

Ya en el 2007, en el gobierno de Chávez, el Estado venezolano le había

3 La "hegemonía comunicacional" o control de los medios de comunicación es un concepto que fue ampliamente usado por el presidente Hugo Chávez en Venezuela para referirse a lo que él percibía como una necesidad para la Revolución Bolivariana. En el 2007, se convirtió en una meta explícita de su gobierno y fue incluida en el Proyecto Nacional Simón Bolívar, equivalente al Plan de la Nación. 
retirado la concesión a Radio Caracas Televisión (RCTV). Sin embargo, el nuevo modelo implantado por Maduro fue menos agresivo que el de su predecesor: consistió en la compra de los medios de comunicación con línea editorial neutra o favorable a la oposición. La adquisición no era forzosa, aunque sí se presionaba a ella por vías tributarias o de otra naturaleza, y se pagaba a precios elevados (Reyes, 2013). La inviabilidad del medio vendido no tenía que ver con un mal modelo de negocios, sino que era consecuencia de las sanciones directas e indirectas que imponía el régimen (Cañizales y Matos-Smith, 2015). De esta manera, el gobierno del presidente Maduro logró hacerse con un entramado de medios y el control oligopólico de la información, y con el color rojo de la Revolución Bolivariana como orientación editorial.

\section{Pero audiencias azules...}

Fue distinto lo que ocurrió con las audiencias. Los medios pueden comprarse y venderse en el mercado libremente, pero las audiencias no se transan con la misma facilidad, y asumieron posturas que se rebelaban al rojo editorial. El reporte de audiencia televisiva de la encuestadora Nielsen levantado entre el $11 \mathrm{y}$ el 15 de agosto del 2013 mostró que cinco televisoras privadas concentraban casi la mitad de la audiencia nacional ${ }^{4}$, mientras que las cinco televisoras públicas nacionales tenían apenas $8 \% 5$.

El Estado venezolano falló en su esfuerzo por conquistar a las audiencias. Tras la revocación de la concesión de RCTV en el 2007, que fuera el canal favorito de la teleaudiencia venezolana, y la confiscación de sus equipos, los números de audiencia del canal sucesor (TVes) mermaron de manera notable: del $46 \%^{6}$ a menos del $2 \%$. El fracaso en conquistar audiencias propias devino en el uso indiscriminado de las cadenas de radio y televisión. El presidente Chávez hablaba en cadena nacional de radio y televisión más de cien horas al año. Un ritmo aún más intenso siguió su discípulo Nicolás Maduro, con un promedio de media hora diaria, según lo reporta el seguimiento de la ONG Monitoreo Ciudadano (s. f.).

Con el incremento de las cadenas de radio y $\mathrm{TV}$, las operadoras de cable del país encontraron un importantísimo incentivo a las ventas de canales de pago. La industria de la televisión por cable en Venezuela creció a un ritmo intenso, al punto que hoy dos tercios de los hogares urbanos tienen acceso a la televisión por suscripción, libre de las cadenas nacionales. Tras el diseño de esa nueva

4 Venevisión, Televén, Globovisión, Meridiano y La Tele.

5 VTV, TVes, Telesur, Asamblea Nacional TV y Vive TV.

6 Penetración del 46 \% de la audiencia (AGB) y un share del 28,1 \% (Cañizales y Matos-Smith, 2015, p. 128). 
arquitectura comunicacional y la hegemonía del mensaje consolidada durante el segundo semestre del año 2013, la penetración de la televisión por cable aumentó 11 puntos. De un 55,75 \% o 1177748 hogares con el servicio alternativo a la TV de señal abierta, hasta más de las dos terceras partes del país, un $66,86 \%$, equivalente a 1390608 hogares (Latin American Mutichannel Avertising Council, 2015) ${ }^{7}$.

Luego del fracaso en los números de audiencia durante el gobierno de Chávez, el nuevo modelo de ocupación de los medios prometía mejorar. Sin embargo, también los medios controlados desde la presidencia de Maduro experimentaron una disminución constante en los afectos de las audiencias. La ya mencionada medición de audiencia de Nielsen de agosto del 2013, después de la venta de Globovisión, le otorgó al canal solo el 3,18 \%, cuando solía estar alrededor del $8 \%$, doblando esa cifra en períodos electorales.

Pero no solamente las audiencias televisivas migraron a la TV paga por cable. También hubo tropismos internos en los medios digitales. Ambos fenómenos concuerdan con lo identificado por Iyengar y Hahn (2009): hay una creciente evidencia de que en el nuevo esquema de medios los electores identifican sesgos en los canales informativos y buscan fuentes alternativas de información que sean más coincidentes con sus preferencias políticas. La existencia de opciones alternativas de información hace posible que las audiencias contrasten las fuentes informativas y decidan cambiar de canal o migrar de plataforma. El desarrollo de la televisión por cable y la existencia de numerosas opciones informativas en internet crean un medioambiente informativo mucho más fragmentado y competitivo que el que podría haber existido veinte años atrás, lo que le brinda al lector/espectador un abanico mucho más amplio para escoger sus fuentes. En el caso venezolano, a partir del punto de inflexión del 2013 se generó una eclosión de nuevos medios digitales. Algunos de ellos derivaron de medios en papel, y otros de la expulsión o renuncia de comunicadores a los medios que cambiaron su línea editorial. RunRun.es, Tal Cual Digital, Efecto Cocuyo, El Pitazo, Caraota Digital, El Estímulo y Armando.info son algunos buenos ejemplos del nuevo periodismo digital venezolano.

Con respecto a los portales de noticias en internet, el que durante años había sido el más visitado de Venezuela, Noticias24. com, sufrió una merma importante luego del cambio de su línea editorial, siendo alcanzado por otro portal mucho más joven: LaPatilla.com, que hasta la fecha se ha mantenido como puntero de los medios digitales. La figura 3 es concluyente al respecto. Otro tanto ocurrió con los medios impresos y su presencia digital. Durante el segundo semestre del año

7 Penetración de TV paga. 
2013, las audiencias dieron un vuelco muy importante. El portal de Últimas Noticias era el claro líder del segmento de presencia digital de medios impresos a mediados de julio del 2013. Un par de meses después de que se hiciera pública la venta del medio, fue destronado por eluniversal.com. Para finales de año, también lo había alcanzado el-nacional.com.

\section{Figura 3. Evolución del ranking en internet de diarios y portales de noticias venezolanos} (del 30 de julio del 2013 al 20 de enero del 2014)

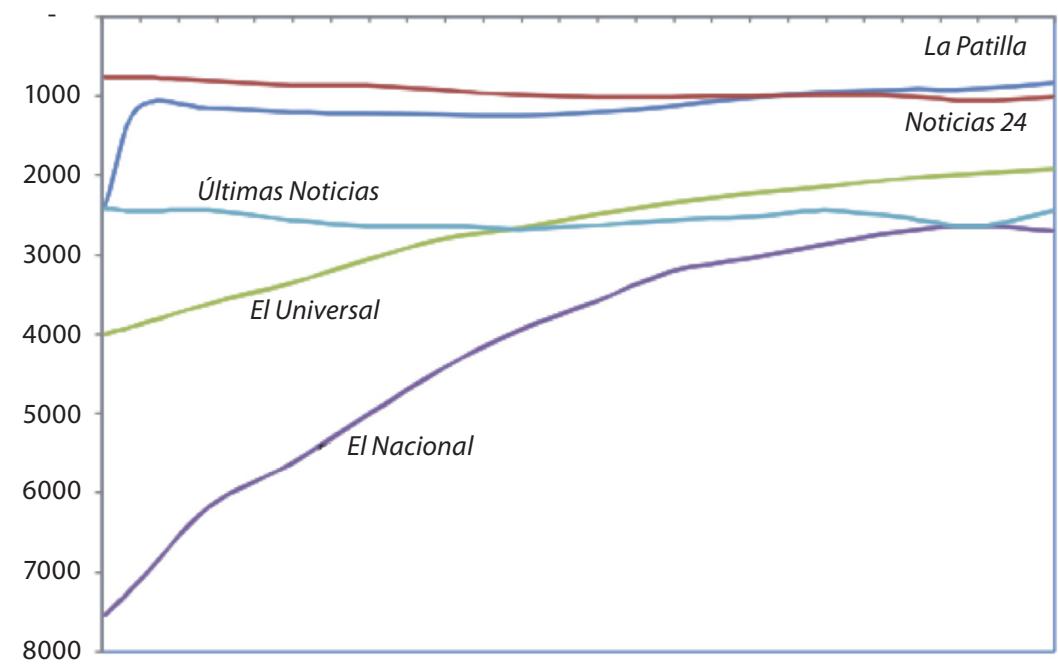

Elaboración propia con datos de Alexa (alexa.com) sobre el ranking relativo de los sitios en internet. Las cifras corresponden a las posiciones relativas de los sitios en el ciberespacio (las mejores posiciones están representadas por los números menores).

La figura 3 muestra cómo durante ese semestre, tras la consolidación de la hegemonía mediática, se cruzan las líneas de los cinco principales medios, lo que muestra la decisión deliberada de las audiencias de no acompañar esos cambios editoriales de línea oficialista. Aun así, la tan ansiada hegemonía comunicacional que logró el gobierno cuando empezaba a ser minoría política en la sociedad tuvo un impacto, sin duda. La nueva arquitectura de medios en Venezuela facilitó al gobierno su control y la orientación de la agenda noticiosa nacional; sin embargo, el control comunicacional fue insuficiente para recuperar las simpatías políticas.

\section{La elección parlamentaria del 2015: un hito}

En este entorno de hegemonía comunicacional y de estatización de la información, se convocaron las 
elecciones parlamentarias del 6 de diciembre del 2015. De poco valió en este sentido la estatización del espectro de medios y el control del mensaje, pues fueron unas elecciones en las que se produjo un cambio sustantivo en las preferencias políticas de los votantes. Con una participación del 74,17\% del electorado, un incremento del 7,7 \% respecto a las legislativas del 2010, las elecciones a la Asamblea Nacional dieron a la oposición venezolana una importante victoria. La Mesa de la Unidad Democrática (MUD), principal movimiento de oposición al presidente Nicolás Maduro, alcanzó 112 diputados de los 167 que componen la Asamblea Nacional. Con el 56,2 \% de la votación nacional, la oposición se hizo con el $72 \%$ de la representación parlamentaria y cerró el largo ciclo de victorias electorales del chavismo que había durado 17 años y más de una docena de elecciones.

La campaña y la elección se topaban con un obstáculo que parecía difícil de salvar: el monopolio de las comunicaciones por parte del gobierno. Es en ese marco donde se idea el canal de YouTube como plataforma fundamental de las comunicaciones de la Unidad Democrática, la plataforma opositora que agrupa a más de una docena de partidos políticos venezolanos. Al respecto, María Fernanda Flores ${ }^{8}$, quien estuvo a cargo del proyecto, cuenta:
Esta idea fue de Chúo Torrealba, coordinador de la Mesa de la Unidad Democrática. Se dividieron las áreas. Se contrató una productora para montar una plataforma comunicacional que generara contenidos. Era un gran rompecabezas que me tocó armar, poner al aire y armar la programación.

De esta manera, la plataforma se convirtió en una alternativa a la voz oficial. La noche de la elección, llegó a un pico culminante en sus niveles de audiencia, tras la ansiedad de una larga demora en la entrega de resultados.

El canal de YouTube alcanzó amplias audiencias, superiores en repetidos momentos a los de los canales de TV de señal abierta. Así, se convirtió en una importante alternativa informativa a la de la voz oficial. "Tuvimos picos de 90000 personas viendo la transmisión en vivo", asegura María Fernanda Flores. El usuario promedio del canal Sala de Prensa Unidad era un hombre (67\%), entre 25 y 44 años (22\%), y que accedía predominantemente desde Venezuela (67\%).

Ya en las estadísticas de los usuarios se hace clara en este momento la creciente importancia demográfica que va adquiriendo la diáspora (figura 5), sobre todo la asentada en Estados Unidos (16\%), España (5\%) y Colombia y Canadá (2\%), así como la atención que esta dispensa a la política local. Aún para diciembre del 2015 el éxodo migratorio venezolano era incipiente, pero alcanza mayor proporción en los años siguientes.

8 María Fernanda Flores, en consulta personal por la autora en febrero del 2016. 
Figura 4. Perfil demográfico de los usuarios del canal Sala de Prensa Unidad en YouTube

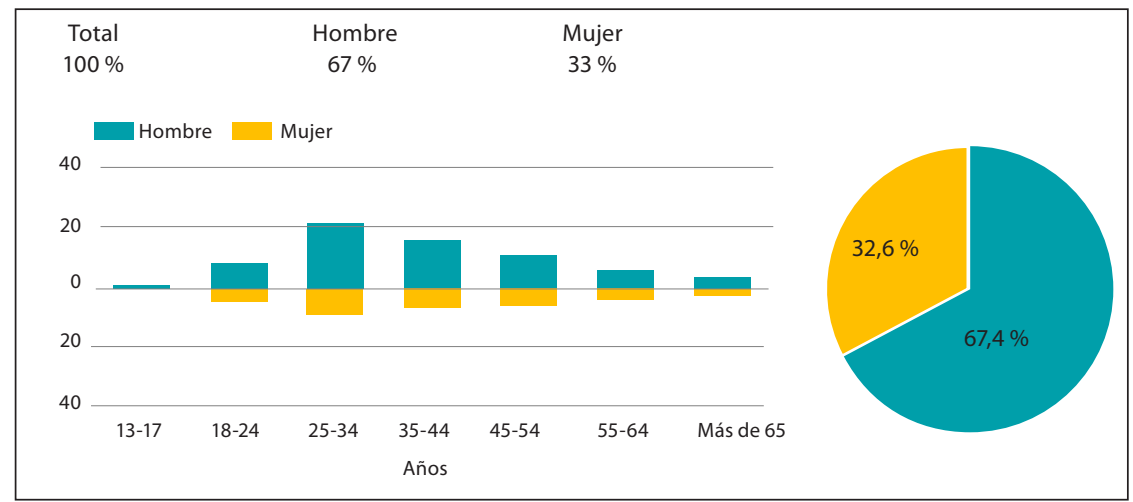

Fuente: Analítica del canal Sala de Prensa Unidad en YouTube, datos suministrados a la autora

Figura 5. Perfil geográfico de los usuarios del canal Sala de Prensa Unidad en YouTube

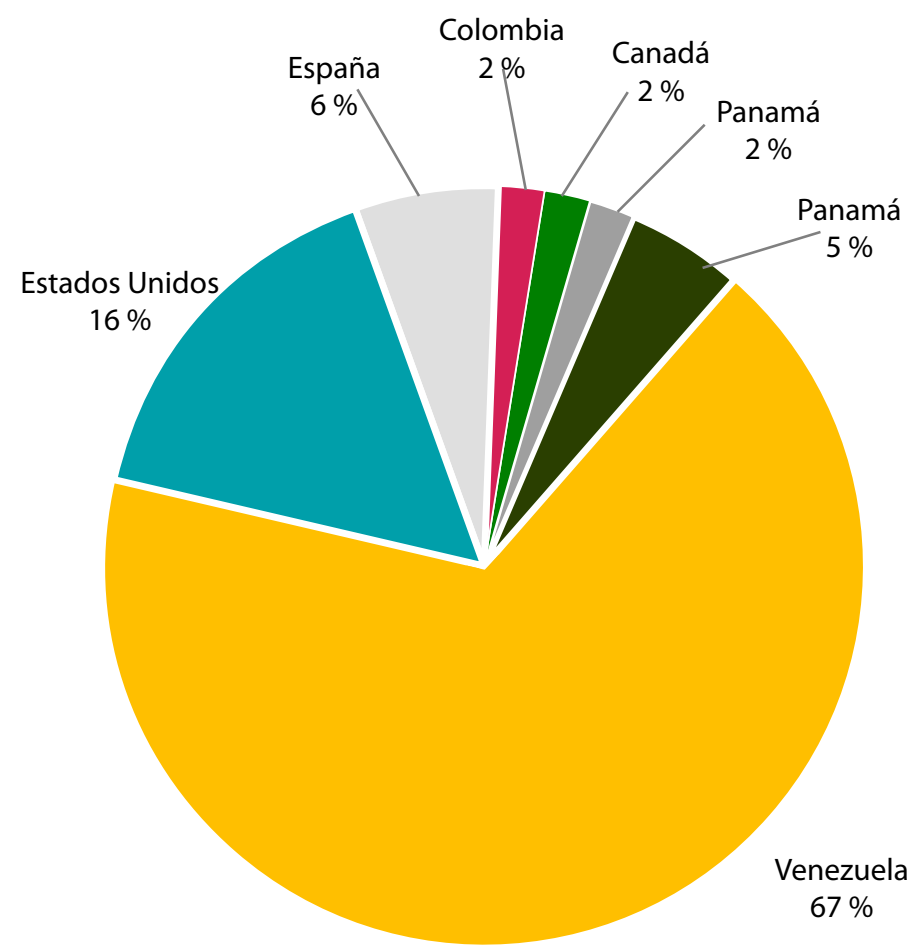

Fuente: Analítica del canal Sala de Prensa Unidad en YouTube, acceso facilitado a la autora 
Ya desde el 2007, Venezuela era el país de América Latina con el uso más intenso de la red como instrumento de activismo político (Fernández, 2008).

El ciberciudadano emplea la red como prótesis de libertad: para paliar sus carencias, sea en su capacidad de acción política o en sus posibilidades de obtener información libre, en sociedades con libertad de prensa disminuida. (Fernández, 2010, p. 47)

El $95 \%$ de los usuarios de internet venezolanos están en Facebook: unos 12 millones de usuarios locales, si bien en términos absolutos esto representa solo el $62 \%$ de la población total. Es menos importante, comparativamente, el uso de YouTube, que alcanza el $38 \%$ de la población, pues las limitaciones del ancho de banda de los proveedores del servicio han restringido el crecimiento de los usuarios de esta plataforma.

Venezuela es puntera también en el uso de Twitter en la subregión, estando a la cabeza de la lista con un $28 \%$ de penetración (Latinobarómetro, 2016), seguida por Ecuador con el $20 \%$. Estos datos del muy alto consumo en redes sociales en Venezuela explican, al menos parcialmente, los elevados números de audiencia que obtuvo el canal de la Unidad en YouTube.
Una vez transcurrida la elección parlamentaria, se indagó entre los electores, en una encuesta de alcance nacional, cuál había sido el medio de comunicación más empleado para obtener información político-electoral durante la campaña (DatinCorp, 2016).

Las respuestas mostraron que la TV seguía siendo el medio principal escogido por los electores para informarse políticamente; el $26 \%$ de ellos lo hacía a través de la TV de señal abierta, cuyos contenidos son controlados en mayor o menor medida por el gobierno de Maduro, como ya hemos analizado antes.

Sin embargo, un bloque similar (23\%) lo hacía a través de canales de cable, o TV por suscripción, lo que incluía canales de producción extranjera con contenidos de gran interés en la política local, como CNN, TVE y Antena 3. Las redes sociales, las publicaciones en internet y otros ítems de respuesta alcanzaban, en conjunto, otro importante $26 \%$.

Estos datos concuerdan con lo encontrado por otras fuentes. Para el 2016, Latinobarómetro, que incluyó por primera vez la medición de redes sociales en su instrumento, advirtió un retroceso de los medios tradicionales como la radio y los diarios, al tiempo que se evidenciaba el crecimiento de internet como fuente de información política. 
Figura 6. Canal preferido para informarse políticamente en Venezuela en el 2016

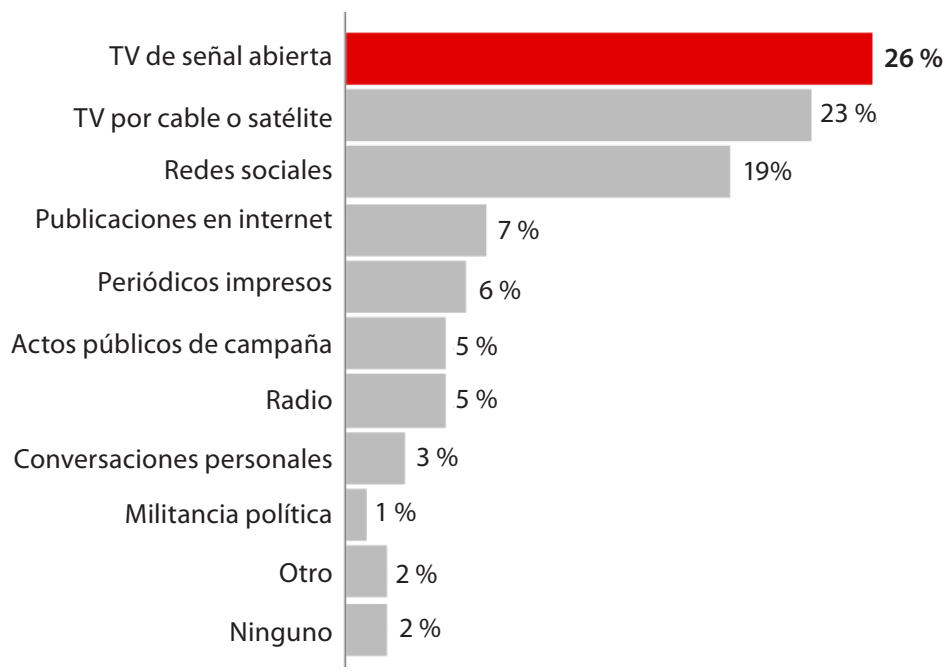

Nota: Pregunta respondida: “Durante las pasadas elecciones parlamentarias, ¿cuál fue el canal que más usó para informarse sobre el proceso electoral?"

Fuente: DatinCorp Venezuela (2016), con verbatim de Carmen Beatriz Fernández

Más interesante aún es analizar los datos de los canales de información política ciudadana cruzados, en función de las preferencias políticas del elector, pues la distribución no es homogénea y varía de acuerdo con las preferencias ideológicas, como ya apuntábamos en un artículo anterior (Rodríguez-Virgili y Fernández, 2017). Al contrastar los datos, encontramos que los canales de
TV se utilizan como fuente informativa por quienes son partidarios del oficialismo. La encuesta DatinCorp (2016) estimó que un $36 \%$ de los partidarios del oficialismo (figura 7) usaron la TV de señal abierta como canal de información política principal, mientras que solo un $17 \%$ de los partidarios de la oposición hicieron lo mismo. En cuanto a las redes sociales como principal medio de

9 Ficha Técnica de la Encuesta DatinCorp (2016). Universo conformado por electores de Venezuela, mayores de 18 años y residentes en el país. Alcance: ciudades. Método: probabilístico tetraetápico (comienza con la selección de las áreas urbanas, luego la de manzanas y calles, la de viviendas y, finalmente, la de los entrevistados); toma en consideración las desagregaciones planteadas en la cobertura. Tamaño de la muestra: 1207 entrevistas. Error máximo muestral esperado de $\pm 2,8 \%$ y nivel de confianza del $95 \%$. Campo en hogares, clasificados por tipo de vivienda, edad y sexo. Fecha: 10 de abril del 2016. 
información política, ocurre exactamente lo contrario: solo un $8 \%$ de los oficialistas tienen en las redes su principal fuente de información política; en cambio, entre los opositores, la cifra se triplica. El $26 \%$ de quienes están en contra del gobierno chavista se informa políticamente a través de redes sociales. En total, un $36 \%$ de los opositores venezolanos encuentran en lo digital su principal fuente de información política (redes sociales y publicaciones informativas en internet), pero únicamente el $12 \%$ de los partidarios del oficialismo afirman lo mismo.

\section{Figura 7. Consumo de medios para la información política en Venezuela en el 2015, discriminado por preferencias políticas}

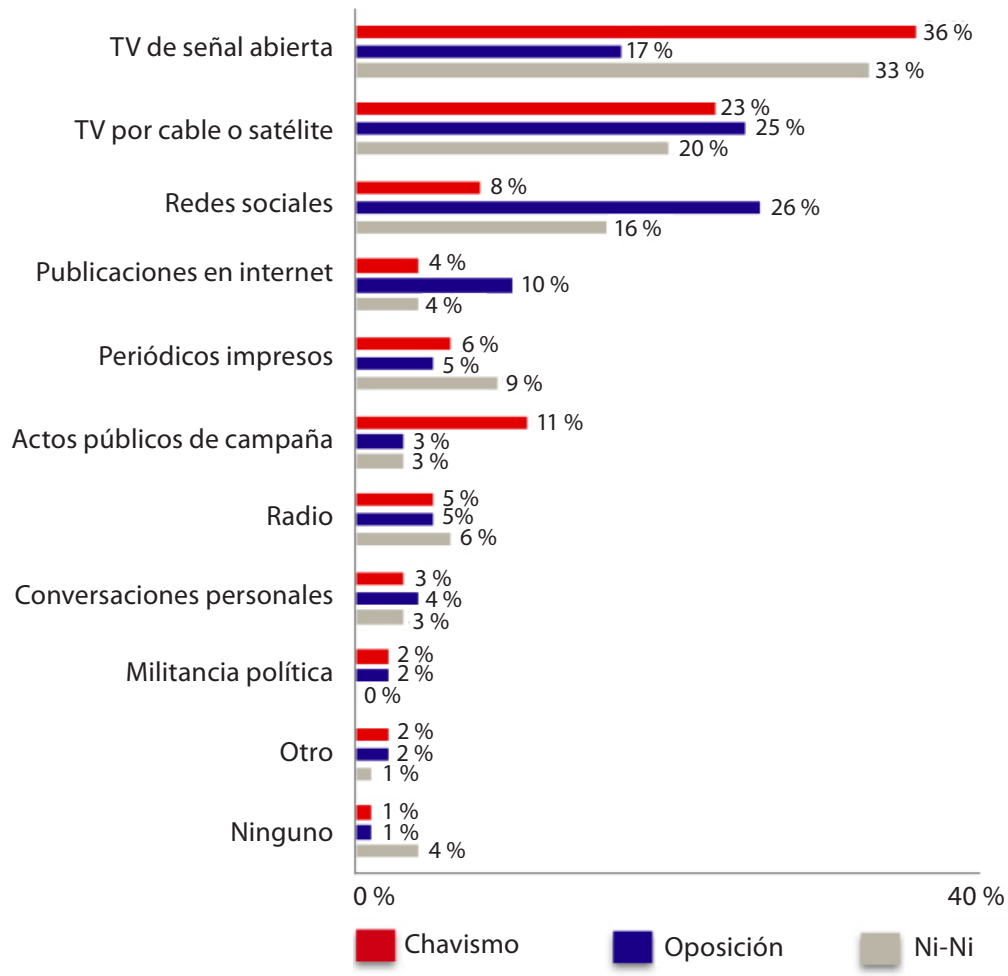

Nota: Pregunta respondida: “Durante las pasadas elecciones parlamentarias, ¿cuál fue el canal que más usó para informarse sobre el proceso electoral?" 
Es decir, también en redes sociales los ciudadanos buscan la información lo más cercanamente posible a sus ideas previas sobre la realidad y se produce una selectividad ideológica. Estos resultados confirman la teoría de la exposición selectiva: el consumo de los medios está determinado por las predisposiciones de la audiencia, que busca confirmar o reforzar sus opiniones en los contenidos de los medios (Lazarsfeld, Berelson y Gaudet, 1948). Esto es, los ciudadanos buscan en la información la mayor consonancia posible con sus ideas previas sobre la realidad (Stroud, 2010). "La teoría predice que, como forma de minimizar la disonancia, la gente busca la información con la que espera estar de

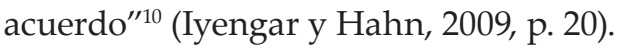

Por temor a ser receptores de propaganda ideológica, las audiencias en Venezuela migraron en todas las plataformas comunicacionales. Este fenómeno es coherente con otros procesos políticos estudiados. La demanda de noticias varía según la percepción de la afinidad con las organizaciones de noticias en las preferencias políticas del consumidor (Iyengar y Hahn 2009). En un ambiente experimental, estos autores encontraron que los electores norteamericanos favorables al Partido Republicano preferían leer reportes de noticias atribuidos a la cadena de noticias Fox y evitaban las noticias de CNN y NPR. Por el contrario, quienes eran afectos al Partido
Demócrata se comportaban exactamente al revés: dividían su atención entre CNN y NPR, pero evitaban las noticias de la cadena Fox. Esta autoselección, basada en la afinidad partidista, se presentaba no solo en la cobertura de noticias de hechos controversiales, sino también con respecto a materias relativamente inocentes como el crimen o los viajes.

\section{¿Tendencia?: la ciberpolítica en el 2018, de la mano con WhatsApp}

Hasta ahora en Venezuela la hegemonía comunicacional no ha podido imponerse sobre la voz ciudadana. Los medios pueden ser muy poderosos para armar la agenda de los temas sobre los que se discute en el país (agenda setting), pero son mucho menos efectivos en poder cambiar las opiniones de los electores y su conducta política.

Sin embargo, y conforme en Venezuela el gobierno de Maduro avanza en la instalación de un proyecto autocrático, nuevas formas de callar las voces no oficiales se han diseñado desde el poder central, y también las redes sociales comienzan a ser penetradas y controladas. Los nuevos medios han ofrecido por ahora una ventana a la libertad y diversidad de información, pero en el 2014 el régimen creó un nuevo Viceministerio de Comunicación para las Redes Sociales, con afanes regulatorios, al mismo tiempo que la

10 Traducción propia del original en inglés. 
represión llegaba a las redes sociales con la encarcelación de varios tuiteros ${ }^{11}$, quizás como mensaje de alerta a toda esta comunidad. En el 2017 se promulgó una ley contra el odio, que pretendió dar soporte a la represión 2.0. Por otro lado, las severas restricciones de divisas que sufren los medios impresos (y, con ello, el limitado acceso al papel) hicieron imposible para varios rotativos de línea independiente seguir en el mercado. Fueron notables los casos de El Carabobeño, Tal Cual y El Impulso. Todo ello ha contribuido a silenciar la visión no oficial del día a día venezolano.

Previendo un nuevo tropismo de las audiencias nacionales, en la búsqueda de formas alternativas de acceso a la información, en una encuesta de alcance nacional reciente indagamos sobre el rol de WhatsApp como medio de información política (DatinCorp, 2017) ${ }^{12}$.

Figura 8. Venezuela: uso de WhatsApp para informarse políticamente en diciembre del 2017

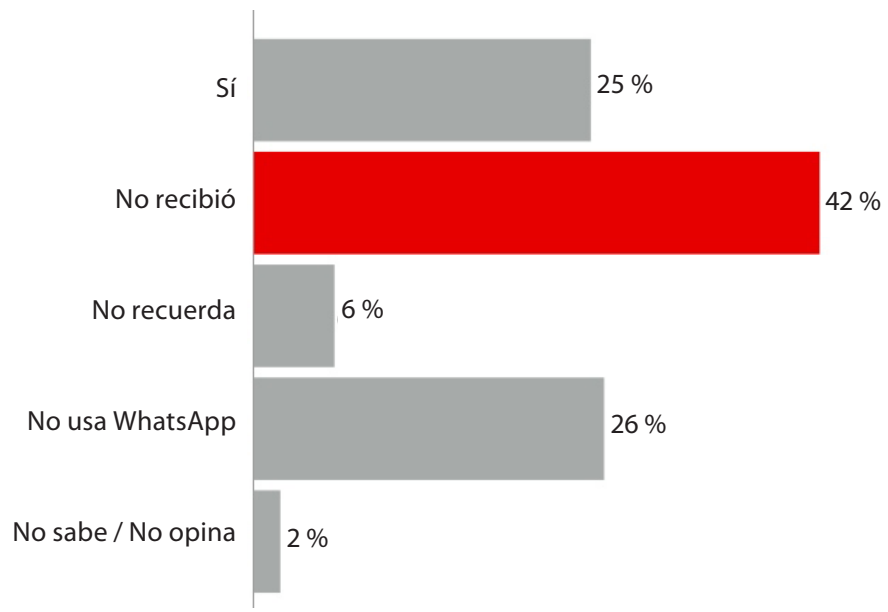

Fuente: DatinCorp Venezuela (2017), encuesta nacional, con verbatim sugerido por Carmen Beatriz Fernández, $n=1,009$

11 Quizás la más conocida sea @inesitaterrible, quien estuvo tres años presa y fue liberada en el 2017 por motivos de salud y, posteriormente, expatriada.

12 Ficha Técnica de la Encuesta DatinCorp (2017). Universo conformado por electores de la República Bolivariana de Venezuela. Alcance: ciudades. Método: probabilístico tetraéptico. Total de electores entrevistados: 1009. Error máximo muestral $\pm 3,1 \%$ y nivel de confianza del $95 \%$. Campo en hogares clasificando a los entrevistados por tipo de vivienda, edad y sexo. Fecha: 17 de diciembre del 2017. 
Uno de cada cuatro venezolanos afirma haber recibido algún mensaje político vía WhatsApp en la semana anterior. Esta cifra ya es en sí misma sorprendente, pero lo es más cuando hacemos el análisis segmentado por la variable de identificación política, pues el fenómeno es mucho más amplio entre quienes se confiesan opositores; en este sector, uno de cada tres electores había recibido información política por WhatsApp en la semana anterior.

Figura 9. Venezuela: uso de WhatsApp para informarse políticamente, por identificación política, en diciembre del 2017

\begin{tabular}{lccccc}
\hline Ítem de respuesta & Total & \multicolumn{3}{c}{ ¿De acuerdo a su definición política, usted se considera? } \\
\cline { 2 - 5 } & $\%$ & CHAVISTA & OPOSITOR & NO ALINEADO & No opina \\
\hline Sí & 249 & 45 & 98 & 96 & 10 \\
& 24,68 & 21,43 & 32,67 & 20,47 & 33,33 \\
No recibió & 422 & 75 & 109 & 230 & 8 \\
& 41,82 & 35,71 & 36,33 & 49,04 & 26,67 \\
No recuerda & 63 & 11 & 24 & 24 & 4 \\
& 6,24 & 5,24 & 21,33 & 8,00 & 13,33 \\
No usa WhatsApp & 259 & 76 & 64 & 113 & 6 \\
& 25,67 & 36,19 & 21,33 & 24,09 & 20,00 \\
No sabe / No & 16 & 3 & 5 & 6 & 2 \\
opina & 1,59 & 1,43 & 1,67 & 1,28 & 6,67 \\
Totales & 1009 & 210 & 300 & 469 & 30 \\
Porcentaje (\%) & 100,00 & 20,81 & 29,73 & 46,48 & 2,97 \\
\hline
\end{tabular}

Los datos son explícitos y sugieren que WhatsApp podría estar siendo el nuevo destino en la migración de plataformas como fuente de información política que vienen experimentando las audiencias venezolanas.

El fenómeno del aumento de la importancia relativa de los nuevos medios es global y viene ocurriendo en todas las sociedades del mundo, con mayor intensidad conforme mayores son los índices de penetración de internet y la familiaridad con las nuevas herramientas. Sin embargo, el fenómeno parece ser más intenso y acelerado en el caso venezolano, donde, además de darse una migración de audiencias desde lo analógico al mundo digital, hay una respuesta deliberada de esas audiencias en rebelión a las ambiciones de control de la información desde el gobierno nacional.

El uso intensivo de las fuentes digitales como medio de información política en Venezuela se relaciona con la 
consolidación de la llamada "hegemonía comunicacional", en clara consonancia con la teoría de la exposición selectiva. Para Venezuela, los valores encarnados por la red coinciden con la aseveración de Castells (2001): “Internet y libertad son sinónimos".

\section{Referencias}

AGB Nielsen Media Research Venezuela. (2013). Medición de audiencias. Agosto. Informe privado.

Cañizales, A., y Matos-Smith, M. (2015). El caso de Globovisión y la implantación del modelo mixto-autoritario en el sistema de medios. Revista Iberoamericana, 15(59), 127-140.

Castells, M. (2001). Internet, libertad y sociedad: una perspectiva analítica. Recuperado de http://www.uoc.edu/web/esp/launiversidad/inaugural01/intro_conc.html.

DatinCorp. (2016). Estudio de coyuntura política. Venezuela. Abril 2016.

DatinCorp. (2017). Crisis y desenlaces. Diciembre 2017.

Fernández, C. B. (2010). Tres años husmeando en la ciberpolítica latinoamericana. Comunicación. Estudios Venezolanos de Comunicación, 150(2), 42-48.

Fernández, C. B. (2008). Ciberpolítica: ¿cómo usamos las tecnologías digitales en la política latinoamericana? Buenos Aires: Konrad-Adenauer-Stiftung.

Fernández, C. B. (2014). Venezuela 2014 y la neonata arquitectura comunicacional. Revista IESA. Recuperado de http://virtual.iesa.edu.ve/servicios/wordpress/ wp-content/uploads/2016/04/2014-1-carmenbeatriz.pdf

Gallup. (s. f.). Presidential Approval Ratings: Gallup Historical Statistics and Trends. Recuperado de http://news.gallup.com/poll/116677/presidential-approval-ratings-gallup-historical-statistics-trends.aspx

Gallup. (2017). Presidential Approval Ratings: Barack Obama. Recuperado de http:// news.gallup.com/poll/116479/barack-obama-presidential-job-approval.aspx

Internet World Stats. (s. f.). North America Internet Usage Stats, Facebook and 2018 Population Statistics. Recuperado de https://www.internetworldstats.com/america.htm\#us

Iyengar S., y Hahn, K. (2009). Red Media, Blue Media: Evidence of Ideological Selectivity in Media Use. Journal of Communication, 59, 19-39.

Latin American Multichannel Advertising Council. (2015). Métricas de TV paga en Venezuela: Penetración de TV paga en hogares 2007-2015. Recuperado de https:// web.archive.org/web/20170425045300/http://www.lamac.org/venezuela/metricas/total-por-tv-paga 
Latinobarómetro. (2016). Uso de redes sociales. Recuperado de http://www.latinobarometro.org

Lazarsfeld, P. F., Berelson, B. R., y Gaudet, H. (1948). The People's Choice. Nueva York, NY: Columbia University Press.

Monitoreo Ciudadano. (s. f.). caden-ó-metro: Exigimos que termine el abuso. Mira el contador de horas de Maduro. Recuperado de www.monitoreociudadano.org/cadenometro/

Reyes, L. M. (10 de junio del 2013). La venta de los medios en Venezuela. Politikom. Recuperado de http://politikom.wordpress.com/2013/06/10/la-venta-de-los-medios-en-venezuela/

Rodríguez-Virgili, J., y Fernández, C. B. (2017). Infopolítica en campañas críticas: el caso de Argentina, España y Venezuela en 2015. Comunicación y Hombre, 13, 85-102.

Sala de Prensa Unidad. (6 de diciembre del 2015). EN VIVO 6 de Diciembre [archivo de video, actualmente no disponible]. Recuperado de https://www.youtube. com/channel/UCLVJxAzxBTbL815OuxQp4mw

Stroud, N. J. (2010). Population and Partisan Selective Exposure. Journal of Communication 60(3), 556-576. https://doi.org/10.1111/j.1460-2466.2010.01497.x

The [Obama] White House. (18 de enero del 2017). President Obama Holds his Final Press Conference [archivo de video]. Recuperado de https://www.youtube.com/ watch?v=1OaWeQ6Sw1Y

YouTube. (27 de septiembre del 2016). With nearly 2 million concurrent viewers and over 3 million live watch hours, first presidential debate breaks political record [mensaje en un blog]. Recuperado de https://youtube.googleblog.com/2016/09/with-nearly2-million-concurrent.html 